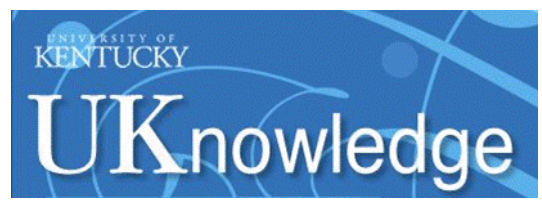

University of Kentucky

UKnowledge

$1-9-2015$

\title{
Design of Organic Ternary Blends and Small-Molecule Bulk Heterojunctions: Photophysical Considerations
}

\author{
Kallarakkal Ramakrishnan Rajesh \\ Oregon State University \\ Keshab Paudel \\ Oregon State University \\ Brian Johnson \\ Oregon State University \\ Rawad Hallani \\ University of Kentucky, rawad.hallani@uky.edu \\ John E. Anthony \\ University of Kentucky, john.anthony@uky.edu
}

See next page for additional authors

Follow this and additional works at: https://uknowledge.uky.edu/chemistry_facpub

Part of the Chemistry Commons

Right click to open a feedback form in a new tab to let us know how this document benefits you.

\section{Repository Citation}

Rajesh, Kallarakkal Ramakrishnan; Paudel, Keshab; Johnson, Brian; Hallani, Rawad; Anthony, John E.; and Ostroverkhova, Oksana, "Design of Organic Ternary Blends and Small-Molecule Bulk Heterojunctions: Photophysical Considerations" (2015). Chemistry Faculty Publications. 48.

https://uknowledge.uky.edu/chemistry_facpub/48

This Article is brought to you for free and open access by the Chemistry at UKnowledge. It has been accepted for inclusion in Chemistry Faculty Publications by an authorized administrator of UKnowledge. For more information, please contact UKnowledge@lsv.uky.edu. 


\title{
Design of Organic Ternary Blends and Small-Molecule Bulk Heterojunctions: Photophysical Considerations
}

\author{
Digital Object Identifier (DOI) \\ http://dx.doi.org/10.1117/1.JPE.5.057208
}

\section{Notes/Citation Information}

Published in Journal of Photonics for Energy, v. 5, no. 1, article 057208, p. 057208-1 through 057208-15.

Copyright 2015 Society of Photo Optical Instrumentation Engineers. One print or electronic copy may be made for personal use only. Systematic reproduction and distribution, duplication of any material in this paper for a fee or for commercial purposes, or modification of the content of the paper are prohibited.

Kallarakkal Ramakrishnan Rajesh, Keshab Paudel, Brian Johnson, Rawad Hallani, John Anthony, Oksana Ostroverkhova, "Design of Organic Ternary Blends and Small-Molecule Bulk Heterojunctions:

Photophysical Considerations," Journal of Photonics for Energy, Volume 5, Number 1, Article 057208, 2015. DOI: http://dx.doi.org/10.1117/1.JPE.5.057208

Authors

Kallarakkal Ramakrishnan Rajesh, Keshab Paudel, Brian Johnson, Rawad Hallani, John E. Anthony, and Oksana Ostroverkhova 


\title{
Design of organic ternary blends and small-molecule bulk heterojunctions: photophysical considerations
}

\author{
Kallarakkal Ramakrishnan Rajesh, ${ }^{\text {a }}$ Keshab Paudel, ${ }^{\mathrm{a}}$ Brian Johnson, ${ }^{\mathrm{a}}$ \\ Rawad Hallani, ${ }^{b}$ John Anthony, ${ }^{b}$ and Oksana Ostroverkhova ${ }^{a}, *$ \\ ${ }^{\mathrm{a}}$ Oregon State University, Department of Physics, 301 Weniger Hall, Corvallis, \\ Oregon 97331, United States \\ ${ }^{\mathrm{b}}$ University of Kentucky, Department of Chemistry, 027 Chemistry-Physics Building, \\ Lexington, Kentucky 40506, United States
}

\begin{abstract}
We explored relationships between photophysical processes and solar cell characteristics in solution-processable bulk heterojunctions (BHJs), in particular: (1) polymer donor:fullerene acceptor:small-molecule (SM) nonfullerene acceptor, (2) polymer donor:SM donor:SM nonfullerene acceptor, and (3) SM donor:SM nonfullerene or fullerene acceptor. Addition of a nonfullerene SM acceptor to "efficient" polymer:fullerene BHJs led to a reduction in power conversion efficiency (PCE), mostly due to decreased charge photogeneration efficiency and increased disorder. By contrast, addition of an SM donor to "inefficient" polymer:SM nonfullerene acceptor BHJs led to a factor of two to three improvement in the PCE, due to improved charge photogeneration efficiency and transport. In most blends, exciplex formation was observed and correlated with a reduced short-circuit current $\left(J_{\mathrm{sc}}\right)$ without negatively impacting the open-circuit voltage $\left(V_{\text {oc }}\right)$. A factor of $\sim 5$ higher PCE was observed in SM donor:fullerene acceptor BHJs as compared to SMBHJs with the same SM donor but nonfullerene acceptor, due to enhanced charge carrier photogeneration in the blend with fullerene. Our study revealed that the HOMO and LUMO energies of molecules comprising a blend are not reliable parameters for predicting $V_{\mathrm{oc}}$ of the blend, and an understanding of the photophysics is necessary for interpreting solar cell characteristics and improving the molecular design of BHJs. (C) 2015 Society of PhotoOptical Instrumentation Engineers (SPIE) [DOI: 10.1117/1.JPE.5.057208]
\end{abstract}

Keywords: organic solar cells; photophysics; exciplex; ternary blends; small-molecule bulk heterojunctions.

Paper 14061SS received Sep. 12, 2014; accepted for publication Dec. 5, 2014; published online Jan. 9, 2015.

\section{Introduction}

Organic solar cells have been a subject of intensive research. ${ }^{1-3}$ Over the past 10 years, an impressive increase in power conversion efficiency (PCE) has been achieved due to an improved understanding of the physics behind charge carrier photogeneration and transport in organic semiconductors, as well as of useful structure-property relationships. ${ }^{2-6}$ PCEs of $10 \%$ to $12 \%$ have been demonstrated in several organic donor-acceptor (D/A) systems. ${ }^{1}$ Currently, most successful organic solar cells utilize polymer:fullerene bulk heterojunctions (BHJs) ${ }^{4}$ or vacuum-deposited small-molecule (SM)-based multilayer films. ${ }^{7}$ Solution-processable SMBHJs, as well as polymer-based BHJs with nonfullerene acceptors, are still lacking in performance, ${ }^{8-11}$ although considerable progress has been made in these systems as well. ${ }^{12,13}$ For example, the best SMBHJs currently exhibit PCEs of $\sim 9 \%,{ }^{14}$ and PCEs of $10.1 \%$ have been demonstrated in solution-deposited tandem SM cells. ${ }^{15}$ However, the quest for molecules which would yield more efficient SMBHJs remains open.

Another promising direction which may lead to considerable improvements in solution-processable solar cell PCEs is organic ternary BHJs, in which an additional donor (D) or acceptor (A)

*Address all correspondence to: O. Ostroverkhova, E-mail: oksana@ science.oregonstate.edu

0091-3286/2015/\$25.00 (C) 2015 SPIE

Journal of Photonics for Energy

057208-1

Vol. 5, 2015 
molecule is introduced into the standard, binary blends of donor and acceptor, thus creating $\mathrm{D}_{1}: \mathrm{D}_{2}: \mathrm{A}$ or $\mathrm{D}: \mathrm{A}_{1}: \mathrm{A}_{2}$ blends. ${ }^{16}$ Such additions may allow tuning of open-circuit voltage $\left(V_{\mathrm{oc}}\right)^{17}$ and can enable a variety of processes that may be beneficial for the PCE. These include Förster resonant energy transfer (FRET), cascade charge transfer (CT) ${ }^{18}$ and parallel-like CT. ${ }^{16}$ For example, up to a 38\% PCE enhancement was observed in P3HT:PCBM blends upon addition of $\sim 1 \mathrm{wt} \%$ of a squaraine dye due to FRET that extended the spectral sensitivity of the cell to near-infrared (IR) wavelengths. ${ }^{19}$ Also, increased short-circuit currents $\left(J_{\mathrm{sc}}\right)$ due to additional charge transport pathways and enhanced charge photogeneration enabled by additives to the binary D:A blends were reported in a variety of BHJs. ${ }^{19}$ However, development of efficient ternary blends is a relatively new area, and routes of optimizing such blends to achieve high PCEs are not well understood.

In this paper, we explore solution-processable ternary blends and SMBHJs. In particular, we investigate the following systems: (1) polymer donor (D):fullerene acceptor $\left(\mathrm{A}_{1}\right)$ : SM nonfullerene acceptor $\left(\mathrm{A}_{2}\right),(2)$ polymer donor $\left(\mathrm{D}_{1}\right): \mathrm{SM}$ donor $\left(\mathrm{D}_{2}\right): \mathrm{SM}$ nonfullerene acceptor $(\mathrm{A})$, and (3) SM donor:SM nonfullerene or fullerene acceptor. In blends (1) and (2), we seek to establish the effects of CT state formation and its properties, as well as of cascade CT and/or parallel-like $\mathrm{CT}$, on various solar cell characteristics. Our particular focus is on the relationship between photophysical processes mediated by the additional donor or acceptor molecules and solar cell characteristics. Systems (3) utilize the same SMs as those used in systems (1) and (2), thus providing additional insight into the relationship between D/A interactions and solar cell performance. Since our main focus is on the photophysical picture and on correlations between particular photoinduced interactions and device characteristics, no optimization of device geometry or blend composition and processing conditions to achieve the highest PCEs was performed for any of the blends studied.

\section{Experiments}

\subsection{Materials Considerations}

Materials used in our studies and their HOMO and LUMO energies (obtained from the manufacturer or differential pulse voltammetry $)^{20,21}$ are shown in Fig. 1. Regioregular, electronic grade poly[3-hexylthiophene-2,5-diyl] (P3HT) and [6,6] phenyl C61 butyric acid methyl ester (PCBM) were obtained from Sigma Aldrich and Nano-C, respectively, and were used as received. Synthesis and optical and (photo)conductive properties of functionalized anthradithiophene (ADT) derivatives, ADT-TES-F and ADT-TIPS-CN in Fig. 1, were reported elsewhere. ${ }^{20,22}$ The choice of these ADT derivatives for the present study of ternary blends was

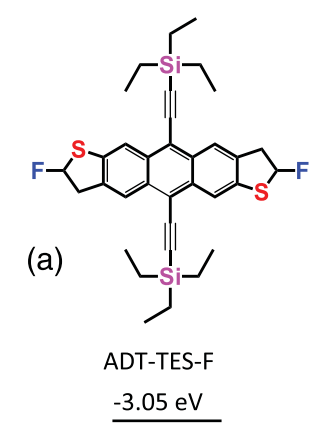

(b)

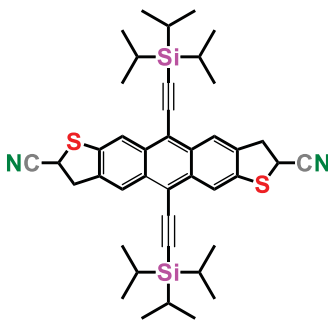

ADT-TIPS-CN

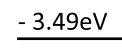

$-5.35 \mathrm{eV}$

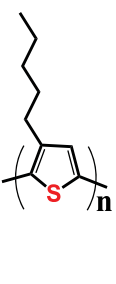

P3HT
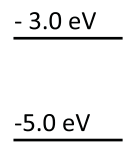

$-5.0 \mathrm{eV}$

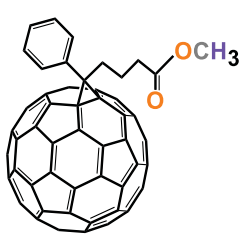

PCBM

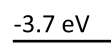

$-5.55 \mathrm{eV}$

Fig. 1 Molecular structures (a) and HOMO and LUMO energies (b) of molecules used in this study. 
Rajesh et al.: Design of organic ternary blends and small-molecule bulk heterojunctions...

guided by the following considerations. Based on the HOMO and LUMO energies of Fig. 1, addition of ADT-TIPS-CN to the P3HT (D):PCBM $\left(\mathrm{A}_{1}\right)$ blend introduces the possibility of cascading CT. By contrast, the energies of ADT-TES-F are similar to those of P3HT, so ADT-TES-F would be expected to act as an additional donor $\left(\mathrm{D}_{2}\right)$ for any ternary blend with P3HT as the main donor, thus enabling parallel-like CT. Furthermore, our previous work revealed that in films containing ADT-TES-F donor and ADT-TIPS-CN acceptor molecules, either efficient FRET from ADT-TES-F to ADT-TIPS-CN occurred or a highly emissive CT state (exciplex) formed, depending on the distance between the $\mathrm{D}$ and A molecules. ${ }^{23}$ This prompted us to explore P3HT $\left(\mathrm{D}_{1}\right)$ :ADT-TES-F $\left(\mathrm{D}_{2}\right)$ :ADT-TIPS-CN $(\mathrm{A})$ ternary blends and, in particular, to investigate the possible effects of FRET and/or exciplex formation on the solar cell characteristics. Finally, we have previously done extensive experimental and computational work aimed to understand time-resolved charge carrier and exciton dynamics in films of pristine ADT-TES-F and its blends with a variety of SM acceptors (including ADT-TIPS-CN and PCBM) using planar interdigitated photodetector-type geometry. ${ }^{20,21,23-28}$ In the present study, we sought to apply insights from our previous work to understanding the performance of these derivatives in solar cells.

\subsection{Fabrication and Characterization of Solar Cells}

Devices were fabricated on cleaned polished float indium tin oxide (ITO, $R_{\mathrm{s}}=20 \Omega / \mathrm{sq}$ )-coated glass. A thin layer $(\sim 40 \mathrm{~nm})$ of poly(3,4-ethylenedioxythiophene)-poly(styrenesulfonate) (PEDOT:PSS) was spun onto ITO substrates at $6000 \mathrm{rpm}$ and dried on a hotplate at $130^{\circ} \mathrm{C}$ for $5 \mathrm{~min}$. Stock solutions of donor and acceptor molecules, as well as additives, were prepared in chlorobenzene at concentrations of $20 \mathrm{mg} / \mathrm{ml}$. Then, solutions of donor, acceptor and additives were mixed at appropriate concentrations to achieve the weight ratio of $1: 1: x$ and sonicated for $1 \mathrm{~h}$. Next, a composite layer was deposited by spin casting the mixture at $1000 \mathrm{rpm}$ and was kept at $100^{\circ} \mathrm{C}$ for $10 \mathrm{~min}$. No high-temperature annealing was performed. Finally, an aluminum (Al) back electrode was deposited by thermal evaporation at $5 \times 10^{-6}$ torr, using a Veeco 7700 evaporator. Several devices were made on each substrate with their area $\left(0.03 \mathrm{~cm}^{2}\right)$ defined using a shadow mask. The cross-sectional structure and bias configurations of the devices are schematically shown in Fig. 2. Current-voltage (I-V) measurements were carried out using a Keithley 237 source-measure unit. For photo I-V measurements, devices were illuminated at $100 \mathrm{~mW} / \mathrm{cm}^{2}$ using a solar simulator (Oriel 96000) with an AM 1.5G filter.

\subsection{Film Structure Characterization}

The structure of resulting BHJ films was assessed in devices by in situ measurements of X-ray diffraction (XRD) using the Bruker D8 XRD system. Figure 3 shows XRD results for out-ofplane structures in ADT-TES-F:PCBM $(1: 1)$, and ADT-TES-F:ADT-TIPS-CN (1:1), and P3HT:PCBM $(1: 1)$ binary blends and in the P3HT:ADT-TES-F:ADT-TIPS-CN $(1: 0.5: 1)$ ternary blend. At room temperature, single ADT-TES-F (ADT-TIPS-CN) crystals have a triclinic structure with unit cell parameters $a=7.12 \AA(8.67 \AA), b=7.23 \AA(10.28 \AA), c=16.63 \AA$ $(12.63 \AA), \alpha=97.52^{\circ}\left(95.52^{\circ}\right), \beta=91.36^{\circ}\left(104.36^{\circ}\right), \gamma=107.49^{\circ}\left(102.48^{\circ}\right) .{ }^{29,30}$ Solutiondeposited pristine ADT-TES-F films exhibit XRD peaks at 5.4, 16.3, and 21.7 deg corresponding

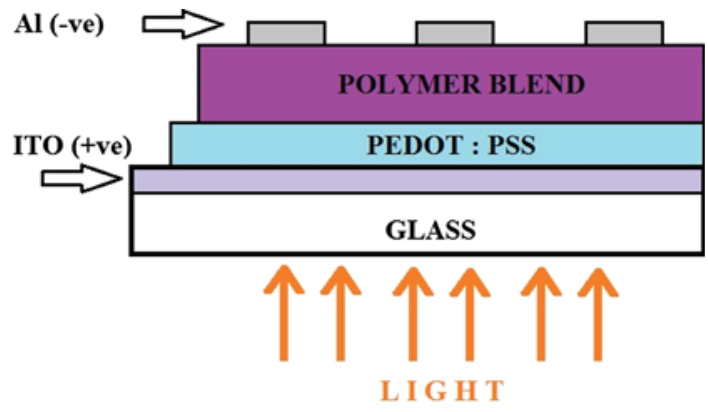

Fig. 2 Device structure schematic. 
Rajesh et al.: Design of organic ternary blends and small-molecule bulk heterojunctions...

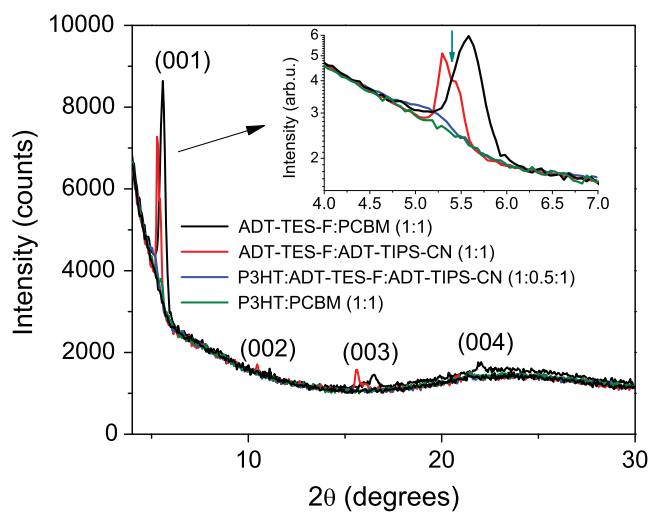

Fig. 3 XRD showing the out-of-plane structure of several binary blends and a ternary blend. Inset shows an expanded view of the 4 to 7 deg region. Peak position corresponding to (001) crystal orientation in pristine ADT-TES-F films is indicated by an arrow.

to the $(00 l)(l=1,3,4)$ orientations, which indicate a crystalline structure with a vertical spacing of $16.6 \AA$ (d-spacing). ${ }^{25,31}$ The (001) crystal orientation, predominant in ADT-TES-F films, corresponds to the molecular $\pi$-planes and the long axis of the conjugated core oriented approximately in the plane of the film, which enables efficient charge transport in ADT-TES-F thin-film-transistors (TFTs). ${ }^{31,32}$ The binary blends of ADT-TES-F with PCBM or with ADT-TIPS-CN acceptors (Fig. 3) preserved the tendency of ADT-TES-F to form crystallites with $(00 l)$ orientations and exhibited co-existence of structures matching those in the pristine ADT-TES-F films and those with the d-spacing slightly modified by the acceptor. In particular, in the ADT-TES-F blend with PCBM (ADT-TIPS-CN), the modified structure was characterized by a d-spacing of $\sim 16 \AA(\sim 17 \AA)$, which is slightly lower (higher) than that in pristine ADTTES-F films. No crystalline domains of PCBM or ADT-TIPS-CN were detected in any binary or ternary blends studied. Likewise, no crystalline order of P3HT was observed in the P3HT:PCBM $(1: 1)$ blend, in agreement with previous studies of such blends prepared using similar conditions, ${ }^{33}$ or in the P3HT:PCBM-based ternary blends. The P3HT:ADT-TIPS-CN $(1: 1)$ binary and P3HT:ADT-TES-F:ADT-TIPS-CN $(1: x: 1)$ ternary blends exhibited a weak feature between 4.8 and $5.3 \mathrm{deg}$ (inset of Fig. 3), which did not change with the ADT-TES-F concentration. This suggests the presence of a small number of ordered P3HT domains with a d-spacing of $\sim 17 \AA$, which would be consistent with the (100) orientation of P3HT crystallites. ${ }^{34}$ No crystalline domains of ADT-TES-F were detected in the P3HT:ADT-TES-F:ADT-TIPS-CN $(1: x: 1)$ ternary blends in the studied range of $x \leq 0.5$.

\subsection{Measurements of Optical and Photoluminescent Properties}

Optical absorption was measured with an Ocean Optics USB-2000 spectrometer using an Ocean Optics LS-1 tungsten halogen lamp as the light source. Photoluminescence (PL) was measured with an Ocean Optics USB-2000FLG spectrometer using a $532 \mathrm{~nm}$ continuous wave (cw) Coherent Verdi-5 laser for excitation. ${ }^{24,25}$ PL spectra were converted to energy units and fit with multiple Gaussian functions as described in our previous paper $^{26}$ to extract peak positions and widths.

PL lifetime measurements were performed via a time-correlated single-photon counting method (PicoQuant TimeHarp 200) using a single-photon avalanche photodiode (Molecular Photonic Devices) for detection. The excitation source was a pulsed $532 \mathrm{~nm}$ laser with a fluence of $20 \mathrm{~nJ} / \mathrm{cm}^{2}$ from Altechna (STA-01-SH-4-MOPA, $470 \mathrm{ps,} 50 \mathrm{kHz}$, Q-switched frequency doubled Nd:YAG). In all PL measurements, a Semrock $532 \mathrm{~nm}$ RazorEdge long-pass filter was used to remove the laser line. To measure the PL lifetimes of specific emission bands, Chroma E625SP and HQ680LP and Thorlabs RG850 filters were used. The data were fit with a single exponential function $[\sim \exp (-t / \tau)$, where $\tau$ is the PL lifetime]. The instrument response function (IRF) $(\sim 0.26 \mathrm{~ns})$ was recorded using scattered light from a frosted glass slide. Most PL measurements were carried out in the same devices as those used in measurements of solar 
Rajesh et al.: Design of organic ternary blends and small-molecule bulk heterojunctions...

cell characteristics. Additionally, optical absorption and PL properties of control samples ADTTES-F:PMMA (1:1) and ADT-TIPS-CN:PMMA (1:1) (PMMA = poly(methyl)methacrylate) were investigated to help in distinguishing between various types of intermolecular interactions in solar cells containing ADT-TES-F and/or ADT-TIPS-CN molecules. Here, PMMA was chosen as a neutral spacer between ADT molecules, having shown no interaction with ADT derivatives in our previous studies. ${ }^{23,27}$

For PL imaging, the samples were placed under an Olympus IX-71 microscope and excited with a 532-nm cw laser through a $40 \times$ objective in the wide-field geometry. Images were obtained using a combination of a 540LP dichroic, 537LP and 695SP or 720LP emission filters (all from Chroma Tech.) and an Andor iXon DU897 EMCCD camera.

\section{Results and Discussion}

\subsection{Optical and PL Properties: Intermolecular Interactions in Binary Blends}

First, we investigated simpler, binary blends in order to identify intermolecular interactions in all intended components for our ternary blends and SMBHJs. Figure 4 shows optical absorption and PL spectra of ADT-TES-F:PMMA (1:1), ADT-TIPS-CN:PMMA (1:1), and ADT-TES-F:ADTTIPS-CN (1:1) films. In ADT-TES-F:PMMA (1:1) films, spectra characterized by a lowestenergy absorption peak at $\sim 547 \mathrm{~nm}(2.27 \mathrm{eV})$ and highest-energy PL emission at $\sim 592 \mathrm{~nm}$ $(2.1 \mathrm{eV})$ were similar to those in pristine ADT-TES-F films, previously attributed to disordered H-aggregates [Figs. 4(a) and 4(b)]. ${ }^{26,27}$ The PL lifetime of ADT-TES-F aggregates in ADT-TESF:PMMA (1:1) films was 1.9 ns [Fig. 4(c)], in agreement with previous studies. ${ }^{26,35}$ Optical absorption of ADT-TIPS-CN:PMMA $(1: 1)$ films was red-shifted as compared to ADT-TESF:PMMA films, with the lowest-energy peak at $\sim 592 \mathrm{~nm}(2.09 \mathrm{eV}){ }^{35}$ PL spectra of ADTTIPS-CN:PMMA films [Fig. 4(b)] had contributions consistent with those of ADT-TIPS-CN

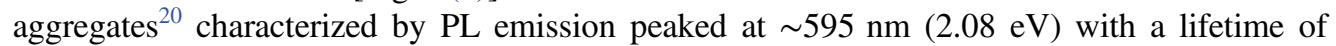
$\sim 0.4 \mathrm{~ns}$ and an excimer emission peaked at $\sim 723 \mathrm{~nm}(1.72 \mathrm{eV})$ with a lifetime of $2.9 \mathrm{~ns}$ [Fig. 4(c)].

Optical absorption of ADT-TES-F:ADT-TIPS-CN (1:1) films was a superposition of the ADT-TES-F and ADT-TIPS-CN absorption spectra [Fig. 4(a)], whereas the PL emission was dominated by that from the ADT-TES-F/ADT-TIPS-CN exciplex peaked at $\sim 691 \mathrm{~nm}$ $(1.80 \mathrm{eV})$ [Fig. 4(b)], with a lifetime of $4.4 \mathrm{~ns}$ [Fig. 4(c)]. CT state formation and its properties in various D:A blends with the ADT-TES-F donor have been previously reported. ${ }^{21,23,24}$ Emissive CT states (exciplexes) were observed when the difference in LUMO energies between the donor and acceptor ( $\triangle \mathrm{LUMO}$ ) was $<0.6 \mathrm{eV}$, which is the case for the ADT-TIPS-CN acceptor. An exciplex with a lifetime of $20 \mathrm{~ns}$ and energy of $1.86 \mathrm{eV}$, exactly matching the energy difference between the HOMO of the ADT-TES-F donor $\left[\mathrm{E}_{\mathrm{HOMO}}(\mathrm{D})\right]$ and the LUMO of the ADT-TIPS-CN acceptor $\left[\mathrm{E}_{\mathrm{LUMO}}(\mathrm{A})\right]$, was observed in ADT-TES-F:ADTTIPS-CN blends with much higher concentrations of the donor than of the acceptor, such as ADT-TES-F:ADT-TIPS-CN (49:1) or (9:1). ${ }^{21,23,36}$ A $0.06 \mathrm{eV}$ down-shift in the exciplex energy (Table 1) and its considerably shorter lifetime in the ADT-TES-F:ADT-TIPS-CN $(1: 1)$ blend, as
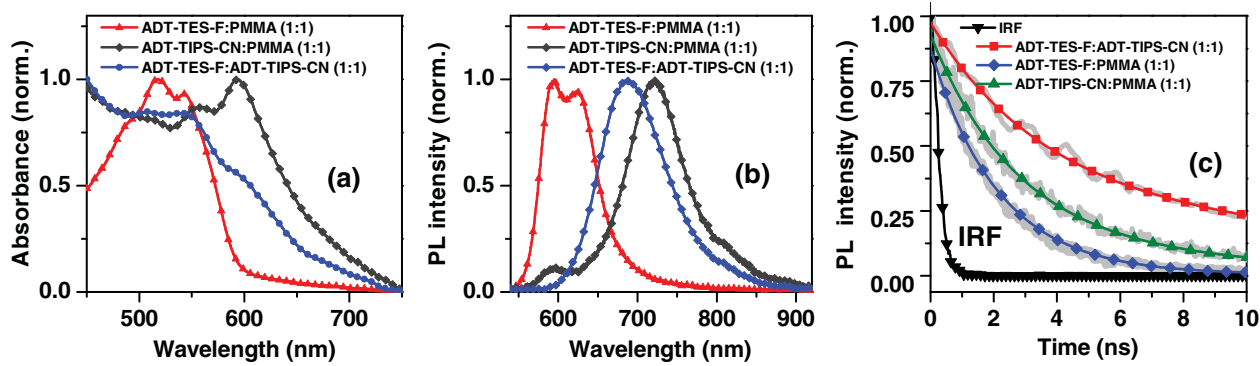

Fig. 4 Normalized optical absorption (a), photoluminescence (PL) spectra (b), and PL lifetime decays (c) of ADT-TES-F:PMMA (1:1), ADT-TIPS-CN:PMMA (1:1), and ADT-TES-F:ADTTIPS-CN (1:1) films. The IRF and exponential fits are also included in (c). 
Table 1 Electrochemical, photophysical, and solar cell parameters of selected bulk heterojunctions (BHJs).

\begin{tabular}{|c|c|c|c|c|c|c|}
\hline Blend & ${ }^{E_{\text {opt.gap,min }}{ }^{a}}$ & $\begin{array}{c}\text { Exciplex } \\
\text { energy } \\
(\mathrm{eV})\end{array}$ & $\begin{array}{c}\mathrm{E}_{\mathrm{LUMO}}(\mathrm{A})-\mathrm{E}_{\mathrm{HOMO}}(\mathrm{D}) \\
(\mathrm{eV})\end{array}$ & $\Delta$ LUMO & $\begin{array}{l}V_{o c} \\
(V)\end{array}$ & $\begin{array}{c}J_{s c} \\
\left(\mathrm{~mA} / \mathrm{cm}^{2}\right)\end{array}$ \\
\hline Р3НТ:РCBM $(1: 1)$ & $1.7^{b}$ & - & 1.3 & 0.7 & 0.6 & 6.9 \\
\hline $\begin{array}{l}\text { P3HT:ADT-TIPS-CN } \\
(1: 1)\end{array}$ & $1.9^{b}$ & 1.40 & 1.51 & 0.49 & 0.8 & 0.016 \\
\hline ADT-TES-F:PCBM $(1: 1)$ & 1.7 & - & 1.65 & 0.65 & 0.15 & 0.52 \\
\hline $\begin{array}{l}\text { P3HT:ADT-TES-F: } \\
\text { ADT-TIPS-CN } \\
(1: 0.5: 1)\end{array}$ & 1.9 & $1.40 ; 1.86^{\mathrm{c}}$ & $1.51 ; 1.86^{c}$ & $0.49 ; 0.44^{c}$ & 0.54 & 0.048 \\
\hline $\begin{array}{l}\text { ADT-TES-F:ADT- } \\
\text { TIPS-CN }(1: 1)\end{array}$ & $2.1^{d}$ & 1.80 & 1.86 & 0.44 & 0.77 & 0.02 \\
\hline $\begin{array}{l}\text { P3HT:ADT-TES-F } \\
(1: 1)\end{array}$ & 1.9 & 1.79 & 1.95 & 0.05 & 0.46 & 0.016 \\
\hline
\end{tabular}

a The lower optical gap energy between the $\mathrm{D}$ or $\mathrm{A}$ in binary blends or the lowest of three molecules in ternary blends.

${ }^{\mathrm{b}}$ From Ref. 37.

${ }^{c}$ The two numbers correspond to energies of $D_{1} / A$ and $D_{2} / A$ exciplexes, $E_{\text {LUMO }}(A)-E_{\text {HOMO }}(D)$ for $D_{1} / A$ and $D_{2} / A$ combinations, and $\Delta L U M O$ between $D_{1}$ and $A$ and $D_{2}$ and $A$.

${ }^{\mathrm{d}}$ From Ref. 21.

compared to asymmetric ADT-TES-F donor-dominated blends, suggests a modified morphology of the D/A interface in the symmetric blends, which promotes energy relaxation and nonradiative recombination of CT states in these SMBHJs.

Optical absorption and PL spectra of pristine P3HT, P3HT:ADT-TIPS-CN (1:1), and P3HT: ADT-TES-F (1:1) films are shown in Figs. 5(a) and 5(b). No significant spectral broadening to achieve photoresponse at longer wavelengths than that with pristine $\mathrm{P} 3 \mathrm{HT}$ was observed in either blend. No low-energy CT states formed between P3HT and ADT molecules were apparent in the absorption spectra. The PL spectra of P3HT:ADT-TIPS-CN (1:1) films were dominated by ADT-TIPS-CN emission at $\sim 580$ to $700 \mathrm{~nm}$ (exhibiting a vibrational mode progression with $0 \rightarrow$ $k$ peaks, where $k=0,1,2),{ }^{26}$ while the P3HT emission was effectively quenched. No ADTTIPS-CN excimer emission, dominant in ADT-TIPS-CN:PMMA (1:1) films (Fig. 4(b)), was observed, which suggests that photoinduced interactions between P3HT and ADT-TIPS-CN molecules dominated those between adjacent ADT-TIPS-CN molecules, thus preventing excimer formation. Indeed, a PL band in the 800 to $950 \mathrm{~nm}$ region with a dominant $\sim 888 \mathrm{~nm}$ peak $(1.4 \mathrm{eV})$ was observed. Such near-IR emission was not present either in P3HT pristine films or in
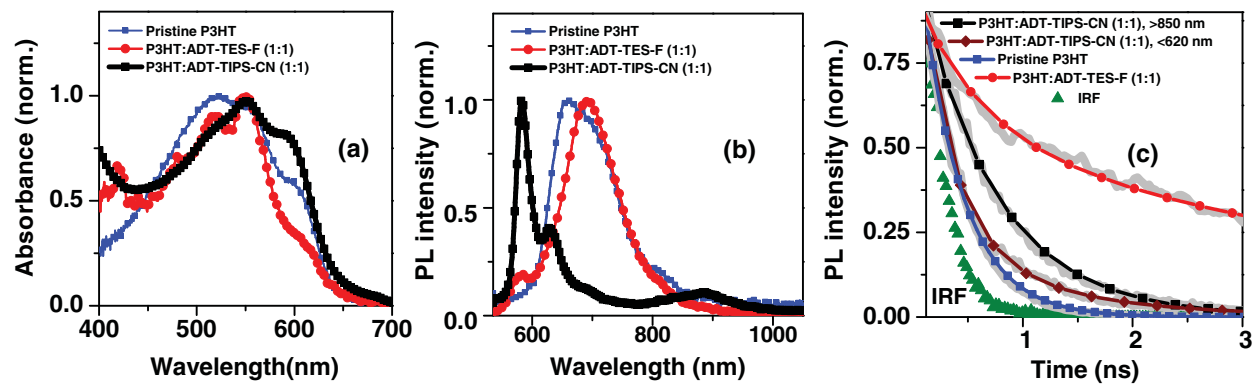

Fig. 5 Normalized optical absorption (a) and PL (b) of pristine P3HT, P3HT:ADT-TES-F (1:1), and P3HT:ADT-TIPS-CN (1:1) films. (c) PL lifetime decays of P3HT:ADT-TES-F (1:1), P3HT:ADTTIPS-CN (1:1) (590-620 nm and 800-950 nm bands separately), and ADT-TES-F:ADT-TIPS-CN (1:1). The IRF and exponential fits are also included in (c). 
Rajesh et al.: Design of organic ternary blends and small-molecule bulk heterojunctions...

ADT-TIPS-CN:PMMA films. To further investigate the origin of this near-IR emission, we separately measured PL lifetimes for the emission at wavelengths $<625 \mathrm{~nm}$ and $>850 \mathrm{~nm}$ [Fig. 5(c)]. The lifetimes yielded 0.34 and $0.69 \mathrm{~ns}$, respectively, which indicate that these emissions originate from different species. Therefore, we assign the $1.4 \mathrm{eV}$ transition, which is $0.11 \mathrm{eV}$ below $\mathrm{E}_{\mathrm{LUMO}}(\mathrm{A})-\mathrm{E}_{\mathrm{HOMO}}(\mathrm{D})$ (Table 1), to an emissive $\mathrm{CT}$ state (exciplex) formed between P3HT and ADT-TIPS-CN.

In P3HT:ADT-TES-F $(1: 1)$ blends, the PL emission was dominated by a transition at $\sim 695 \mathrm{~nm}(1.79 \mathrm{eV})$, with a small residual contribution from the ADT-TES-F aggregate emission peaked at $\sim 590 \mathrm{~nm}$ [Fig. 5(b)]. The dominant band had a PL lifetime of $3.1 \mathrm{~ns}$ [Fig. 5(c)], longer than that of P3HT ( 0.3-0.4 ns) $)^{38}$ or of ADT-TES-F aggregates (1.9 ns). Therefore, we tentatively assign the $1.79 \mathrm{eV}$ emission, which is $0.16 \mathrm{eV}$ below $\mathrm{E}_{\mathrm{LUMO}}(\mathrm{A})-\mathrm{E}_{\mathrm{HOMO}}(\mathrm{D})$ (Table 1), to a P3HT/ADT-TES-F exciplex.

\subsection{Solar Cell Performance and Photophysics}

\subsubsection{Ternary blends}

D: $\mathbf{A}_{1}: \mathbf{A}_{2}$ versus $\mathbf{D}: \mathbf{A}_{1}$ and $\mathbf{D}: \mathbf{A}_{2}-P 3 H T: P C B M: A D T-T I P S-C N(1: 1: \boldsymbol{x})$ versus $P 3 H T$ : PCBM (1:1) and P3HT:ADT-TIPS-CN (1:1). In these experiments, we added ADT-TIPS$\mathrm{CN}$ (Fig. 1 ) at 0 to $5 \mathrm{wt} \%$ concentrations (i.e., $x=0$ to 0.105$)$ to the P3HT:PCBM (1:1) mixture to create a $D: A_{1}: A_{2}$ system and investigate the effects of possible cascading CT (inset of Fig. 6) and other processes on the solar cell characteristics. Figure 6 shows the changes in J-V characteristics upon addition of ADT-TIPS-CN. At our range of ADT-TIPS-CN concentrations, the $V_{\text {oc }}$ decreased from 0.6 to $0.52 \mathrm{~V}$, while the fill factor (FF) increased from 0.49 to 0.54 [Fig. 7(a)] as the concentration of ADT-TIPS-CN increased. The $J_{\mathrm{sc}}$ decreased by a factor of $\sim 2.4$, which led to a similar reduction in the PCE [Fig. 7(b)]. Therefore, a system with beneficial cascading CT was not realized in these blends. When the PCBM acceptor was completely replaced with ADTTIPS-CN, which yielded the binary P3HT:ADT-TIPS-CN $(1: 1)$ blend, the FF was considerably lower, and the $J_{\mathrm{sc}}$ was a factor of $\sim 400$ lower than that in P3HT:PCBM $(1: 1)$ blends, leading to a considerably lower PCE (Table 2). However, the $V_{\text {oc }}$ in the binary P3HT:ADT-TIPS-CN (1:1) blend was $0.8 \mathrm{~V}$, which is $\sim 0.2 \mathrm{~V}$ higher than that in P3HT:PCBM $(1: 1)$ blends.

In order to gain insights into the observed trends, we considered the optical and PL properties of these films. Addition of ADT-TIPS-CN to P3HT:PCBM at low concentrations did not significantly change the optical absorption of P3HT:PCBM films; the absorption spectra of all films were dominated by that of P3HT shown in Fig. 5(a). The PL spectra were a superposition of the P3HT and ADT-TIPS-CN bands shown in Fig. 5(b), and the contribution of ADT-TIPS-CN emission to the overall PL spectrum of the ternary blend increased as the ADT-TIPS-CN concentration increased. No evidence of P3HT and ADT-TIPS-CN interaction, such as exciplex

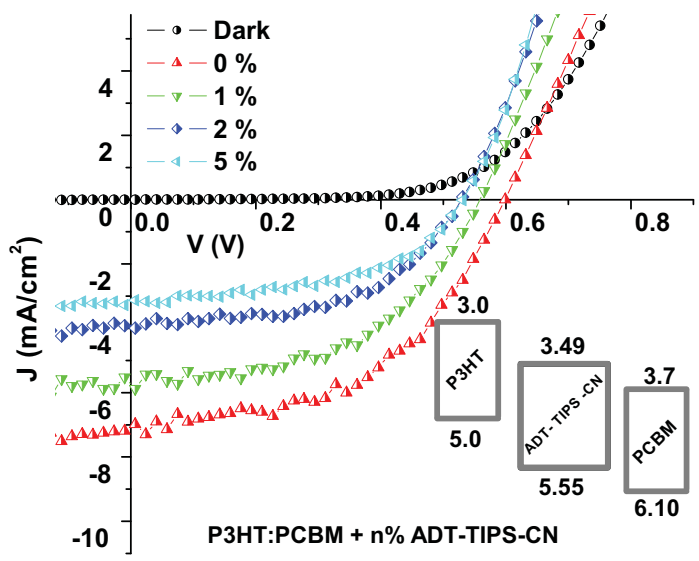

Fig. $6 \mathrm{~J}-\mathrm{V}$ curves obtained in ternary P3HT:PCBM:ADT-TIPS-CN $(1: 1: x)$ solar cells at various concentrations of ADT-TIPS-CN in the 0 to $5 \mathrm{wt} \%$ range ( $x=0$ to 0.1$)$. Dark current in the binary blend $(x=0)$ is also included. Inset shows HOMO and LUMO energies of molecules. 

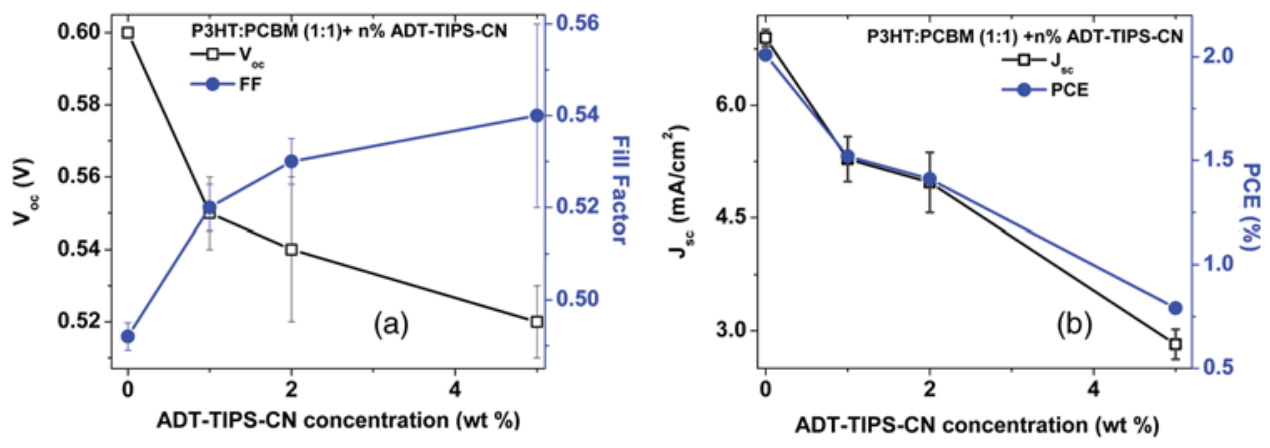

Fig. 7 (a) $V_{\text {oc }}$ (left axis) and FF (right axis) and (b) $J_{\text {sc }}$ (left axis) and PCE (right axis) obtained in ternary P3HT:PCBM:ADT-TIPS-CN $(1: 1: x)$ blends as a function of ADT-TIPS-CN concentration in the 0 to 5 wt \% range $(x=0$ to 0.1$)$. Each value represents an average over four devices, and error bars reflect spread in values.

formation, was observed at 0 to $5 \mathrm{wt} \%$ concentrations of ADT-TIPS-CN. Given the HOMO and LUMO energies of all constituents of the ternary blend (inset of Fig. 6) and no evidence of lowenergy CT states under these conditions, ${ }^{39}$ the observed decrease in $V_{\text {oc }}$ in Fig. 7(a) is unexpected. However, it is possible that the addition of ADT-TIPS-CN increased the density of defects that act as charge traps, which has been known to negatively impact $V_{\text {oc }}{ }^{40}$ Such trap states would also increase charge carrier recombination resulting in a lower $J_{\mathrm{sc}}$, as observed in Fig. 7(b). No negative effect of such increased recombination on the FF was observed [Fig. 7(a)], ${ }^{41}$ which would be consistent with a larger contribution of monomolecular trapassisted recombination (first-order process) than bimolecular recombination (second-order process) into carrier dynamics in the ternary blends as compared to binary P3HT:PCBM blends. ${ }^{18}$

Binary P3HT:ADT-TIPS-CN $(1: 1)$ blends showed considerably lower $J_{\text {sc }}$ values than those in ternary blends, which suggest low charge photogeneration efficiency. Performance of 22 P3HT-based binary BHJs with various acceptors has been summarized in Ref. 42, and it was concluded that a $\triangle$ LUMO of at least $0.2 \mathrm{eV}$ was necessary to achieve photoresponse. In our case, a $\Delta$ LUMO of $0.49 \mathrm{eV}$ was not sufficient to promote efficient charge separation at the D/A interface, which indicates that $\triangle \mathrm{LUMO}$ is not a reliable indicator of photoinduced charge separation. ${ }^{43}$ Instead, exciplex formation was observed [Fig. 5(b) and Table 1], which most likely favors radiative recombination over dissociation into mobile charge carriers, as has been the case in a variety of D:A blends. ${ }^{21,23-25,28}$ This depletes more efficient charge photogeneration pathways, thus lowering the $J_{\mathrm{sc}}$. The low-energy exciplex, however, did not seem to negatively impact the $V_{\mathrm{oc}}{ }^{39}$

$\mathbf{D}_{1}: \mathbf{D}_{2}: \mathbf{A}$ versus $\mathbf{D}_{1}: \mathbf{A}-P 3 H T: A D T-T E S-F: A D T-T I P S-C N(1: x: 1)$ versus P3HT:ADTTIPS-CN (1:1). In these experiments, we added ADT-TES-F (Fig. 1) at 0 to $20 \mathrm{wt} \%$ concentrations (i.e., $x=0$ to 0.5$)$ to the P3HT:ADT-TIPS-CN $(1: 1)$ blend to realize a $\mathrm{D}_{1}: \mathrm{D}_{2}$ :A system, in which ADT-TES-F would act as an additional donor $\left(\mathrm{D}_{2}\right)$. As discussed in Sec. 2.3, the ADT-TES-F derivative cast from solution onto glass substrates forms polycrystalline films with the crystallites predominantly oriented such that their $a-b$ plane is in the plane of the substrate [(001) orientation] ${ }^{25,31,32}$ Such structural arrangement results in strong $\pi-\pi$ overlap in the $\pi$-stacking directions of ADT-TES-F, which explains the good performance of spin-cast ADT-TES-F TFTs, characterized by hole mobilities of $>1.5 \mathrm{~cm}^{2} /(\mathrm{Vs}) .{ }^{31,32}$ Charge transport in the direction of the crystal $c$-axis, which is important for the vertical device geometry of a solar cell, is not expected to be efficient. However, in the ternary blends under study, no ADT-TES-F crystallites were detected by the XRD (Fig. 3), and it is possible that some fraction of ADT-TES-F molecules could form amorphous aggregates ${ }^{27}$ favorable for efficient vertical charge transport. Additionally, pristine ADT-TES-F films exhibit fast charge carrier photogeneration and high photoconductive gains,$^{20}$ which could improve the overall charge photogeneration efficiency in the ternary blends. Finally, in ternary blends, ADT-TES-F and ADT-TIPS-CN molecules may form a FRET pair, as has been observed in ADT-TES-F: ADT-TIPS-CN:PMMA blends, ${ }^{23}$ which could also affect charge carrier photogeneration. 

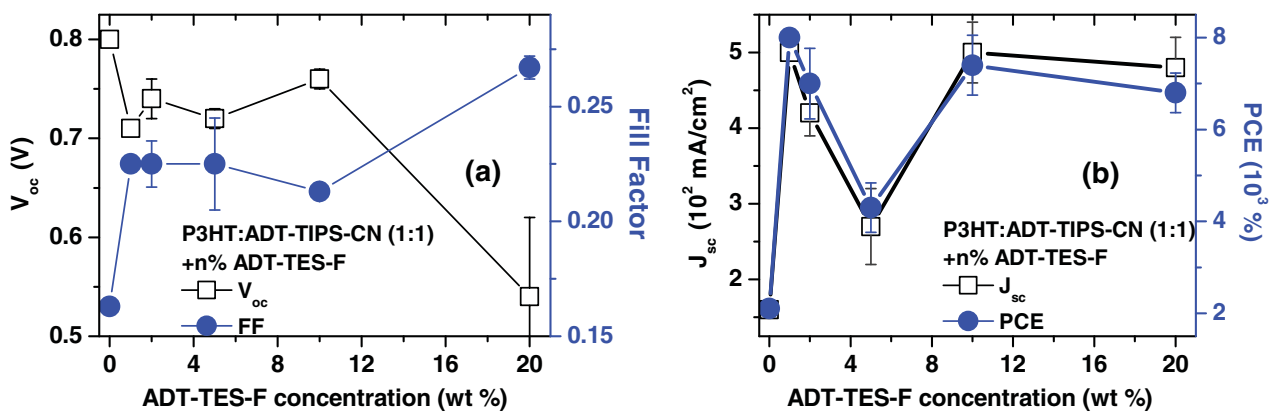

Fig. 8 (a) $V_{\text {oc }}$ (left axis) and FF (right axis) and (b) $J_{\text {sc }}$ (left axis) and PCE (right axis) obtained in ternary P3HT:ADT-TES-F:ADT-TIPS-CN $(1: x: 1)$ blends as a function of ADT-TES-F concentration in the 0 to $20 w t \%$ range $(x=0$ to 0.5$)$. Each value represents an average over four devices, and error bars reflect spread in values.

Figure 8 shows the effects of ADT-TES-F addition to the binary P3HT:ADT-TIPS-CN $(1: 1)$ blend. The $V_{\text {oc }}$ of $\sim 0.8 \mathrm{~V}$ observed in the P3HT/ADT-TIPS-CN blend slightly decreased, most likely due to an increase in charge trap density, ${ }^{40}$ and remained between $\sim 0.72$ and $0.75 \mathrm{~V}$ for ADT-TES-F concentrations of up to $10 \mathrm{wt} \%$. The FF slightly increased, similar to observations in the P3HT:PCBM:ADT-TIPS-CN ternary blends. As the ADT-TES-F concentration further increased, a drop in $V_{\text {oc }}$ was observed, yielding $0.54 \mathrm{~V}$ at $20 \mathrm{wt} \%$ [Fig. 8(a)]. When the ADT-TIPS-CN was completely replaced with ADT-TES-F, in the binary P3HT:ADT-TES-F $(1: 1)$ blend, the $V_{\text {oc }}$ decreased to $0.46 \mathrm{~V}$ (Table 1). As expected, the $J_{\text {sc }}$ improved upon ADT-TES-F addition in the range of 1 to $20 \mathrm{wt} \%$, by a factor of $\sim 2$ to 3 , leading to an improvement in the PCE by a factor of $\sim 2$ to 4 in the ternary blends, depending on the ADT-TES-F concentration, as compared to the binary P3HT:ADT-TIPS-CN $(1: 1)$ blend [Fig. 8(b)]. However, when ADT-TIPS-CN was completely replaced by ADT-TES-F, yielding the binary P3HT:ADT-TES-F $(1: 1)$ blend, the $J_{\text {sc }}$ and PCE were similar to those in the binary P3HT: ADT-TIPS-CN (1:1) blend. Moreover, a considerably lower $V_{\text {oc }}$ in the P3HT:ADT-TES-F $(1: 1)$ blend was observed as compared to that in P3HT:ADT-TIPS-CN $(1: 1)$ (Table 2), a result that cannot be rationalized based on the HOMO and LUMO energies of molecules (Fig. 1).

Next, we sought to relate the above results to photophysics in these films. PL spectra of selected ternary samples are shown in Fig. 9(a). At ADT-TES-F concentrations below $20 \mathrm{wt} \%$, the PL spectra were similar to those of P3HT:ADT-TIPS-CN $(1: 1)$, with varying relative contributions of the 580 to $700 \mathrm{~nm}$ and 800 to $950 \mathrm{~nm}$ bands. The former band is a combination of ADT-TIPS-CN and ADT-TES-F aggregate emission [Fig. 4(b)] and of a residual P3HT emission, whereas the latter band is due to the P3HT/ADT-TIPS-CN exciplex [Fig. 5(b)]. The relative contributions of these two bands to the PL spectra did not exhibit any particular trends with the ADT-TES-F concentration, and so these variations were most likely due to differences in film morphology, as illustrated below. FRET between ADT-TES-F and ADT-TIPS-CN, which should

Table 2 Solar cell characteristics of selected devices.

\begin{tabular}{lccccc}
\hline \hline Blend & Concentration & $V_{\text {oc }}(\mathrm{V})$ & $J_{\mathrm{sc}}\left(\mathrm{mA} / \mathrm{cm}^{2}\right)$ & $\mathrm{FF}$ & PCE $(\%)$ \\
\hline P3HT:ADT-TIPS-CN & $1: 1$ & 0.8 & 0.016 & 0.16 & 0.0021 \\
P3HT:ADT-TES-F & $1: 1$ & 0.46 & 0.016 & 0.22 & 0.0016 \\
ADT-TES-F & pristine & 0.95 & 0.011 & 0.24 & 0.0025 \\
ADT-TES-F:ADT-TIPS-CN & $1: 1$ & 0.77 & 0.02 & 0.35 & 0.0048 \\
ADT-TES-F:PCBM & $49: 1$ & 0.78 & 0.013 & 0.14 & 0.0015 \\
& $3: 1$ & 0.18 & 0.2 & 0.26 & 0.01 \\
& $1: 1$ & 0.15 & 0.52 & 0.28 & 0.024 \\
\hline \hline
\end{tabular}



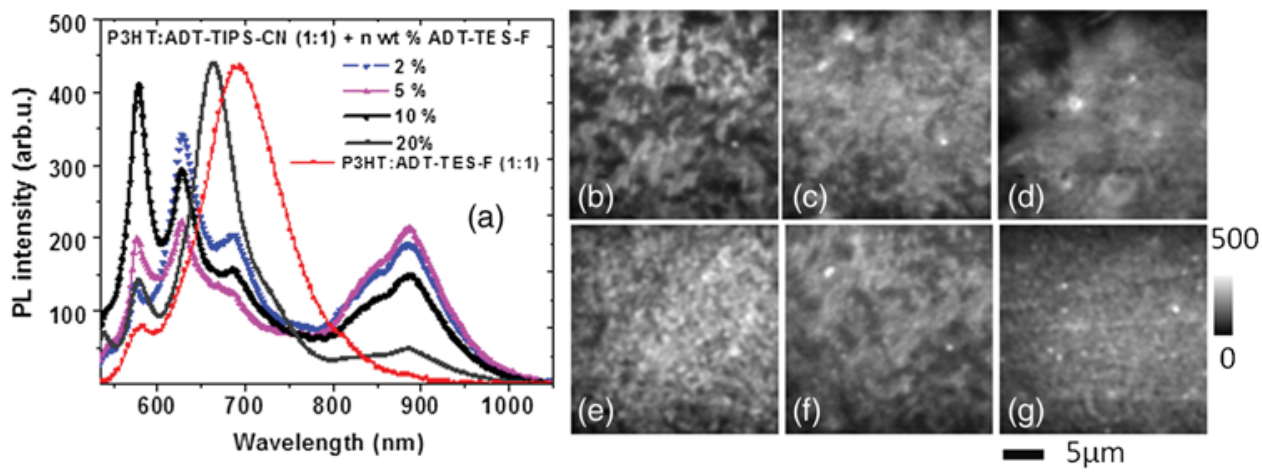

Fig. 9 (a) PL spectra of ternary P3HT:ADT-TES-F:ADT-TIPS-CN blends with various ADT-TES-F concentrations. Spectrum of a binary P3HT:ADT-TES-F blend is also included for reference. (b)(g): PL images of P3HT:ADT-TES-F:ADT-TIPS-CN (1:x:1) films with 2 wt\% [(b) and (e)], 5 wt\% [(c) and (f)], and $20 \mathrm{wt} \%$ [(d) and (g)] of ADT-TES-F. Images in (b)-(d) and in (e)-(g) correspond to $\mathrm{PL}$ emission from the same film areas at $<625 \mathrm{~nm}$ (dominated by ADT-TES-F and ADT-TIPS-CN) and at $>720 \mathrm{~nm}$ (dominated by the P3HT/ADT-TIPS-CN exciplex), respectively.

take place in the ternary blends at sufficiently low ADT-TES-F concentrations,${ }^{23}$ could not be confirmed due to the complexity of PL emission in the ternary blends. However, it is possible that a part of the observed improvement in $J_{\text {sc }}$ in the ternary P3HT:ADT-TES-F:ADT-TIPS-CN blends, as compared to the binary P3HT:ADT-TIPS-CN blends, is mediated by FRET. No CT states formed between P3HT and ADT-TES-F or between ADT-TES-F and ADT-TIPS-CN were apparent at ADT-TES-F concentrations <20 wt $\%$. In P3HT:ADT-TES-F:ADT-TIPS-CN blends with $20 \mathrm{wt} \%$ of ADT-TES-F, the PL emission was dominated by that from the ADT-TES-F/ ADT-TIPS-CN exciplex with the energy of $1.86 \mathrm{eV}$, exactly matching $\mathrm{E}_{\mathrm{LUMO}}(\mathrm{A})-\mathrm{E}_{\mathrm{HOMO}}(\mathrm{D})$ [Fig. 9(a) and Table 1]. ${ }^{23,26}$ Although observation of ADT-TES-F/ADT-TIPS-CN exciplex formation coincided with a dramatic decrease in $V_{\text {oc }}$ in the ternary blends [Fig. 8(a)], these observations are most likely not directly related. Instead, they indicate that a sufficiently high concentration of ADT-TES-F in the ternary blend, such that ADT-TES-F/ADT-TIPS$\mathrm{CN}$ exciplex formation is readily observed, is also high enough to tune the $V_{\mathrm{oc}}$ closer to that in the binary P3HT:ADT-TES-F $(1: 1)$ blend (Table 2).

In order to gain further insight into the variation in $J_{\text {sc }}$ across ADT-TES-F concentrations in the ternary blends [Fig. 8(b)], we performed spectral region-selective PL imaging of P3HT: ADT-TES-F:ADT-TIPS-CN ternary blends containing various ADT-TES-F concentrations. Figures 9(b) to 9(g) show PL images of films with ADT-TES-F concentrations of $2 \mathrm{wt} \%$ [(b) and (e)], $5 \mathrm{wt} \%$ [(c) and (f)], and $20 \mathrm{wt} \%$ [(d) and (g)] obtained from the same film areas at emission wavelengths of $<695 \mathrm{~nm}[(\mathrm{~b})-(\mathrm{d})]$ and $>720 \mathrm{~nm}[(\mathrm{e})-(\mathrm{g})]$. In particular, emission at $<695 \mathrm{~nm}$ is dominated by that from ADT-TIPS-CN and ADT-TES-F, whereas that at $>720 \mathrm{~nm}$ is mostly due to the P3HT/ADT-TIPS-CN exciplex (Sec. 3.1). Therefore, images in Figs. 9(e) to 9(g) reflect the morphology of the interface between P3HT and ADT-TIPS$\mathrm{CN}$ depending on the film. For example, the film with $2 \mathrm{wt} \%$ of ADT-TES-F exhibited ADT-TES-F- and ADT-TIPS-CN-rich (bright) and P3HT-rich (dark) regions [Fig. 9(b)] and extensive networks of P3HT:ADT-TIPS-CN interfaces surrounding sub-micron-size domains of P3HT, ADT-TIPS-CN, and ADT-TES-F molecules not interacting with each other [Fig. 9(e)]. In the film with $5 \mathrm{wt} \%$ of ADT-TES-F, lower PL image contrast was observed [Fig. 9(c)], which suggests more efficient mixing of various components of the ternary blend, resulting in large areas of intermixed P3HT and ADT-TIPS-CN molecules accompanied by large areas of "noninteracting" molecules [Fig. 9(f)]. The PL image of the film with $20 \mathrm{wt} \%$ of ADT-TES-F, in which the PL spectrum at $<695 \mathrm{~nm}$ was dominated by the ADT-TES-F/ADT-TIPS-CN exciplex [Fig. 9(a)], was consistent with efficient mixing between ADT-TES-F and ADT-TIPS-CN molecules [Fig. 9(d)]. However, networks of P3HT/ADT-TIPS-CN interfaces could still be observed [Fig. 9(g)], similar to those in the film with $2 \mathrm{wt} \%$ of ADT-TES-F in Fig. 9(e).

Comparison between the solar cell characteristics of Fig. 8 and the PL data of Fig. 9 suggests that devices with the lowest $J_{\text {sc }}$ [P3HT:ADT-TES-F:ADT-TIPS-CN $(1: 0.1: 1)$, i.e., with $5 \mathrm{wt} \%$ 
Rajesh et al.: Design of organic ternary blends and small-molecule bulk heterojunctions...

ADT-TES-F in Fig. 8(b)] had the highest contribution of the P3HT/ADT-TIPS-CN exciplex into the overall PL spectrum of the film, enabled by efficient intermixing of P3HT and ADT-TIPS$\mathrm{CN}$ in these films [Figs. 9(a) and 9(f), respectively]. Such morphology is not conducive for efficient photoinduced charge separation, in agreement with previous studies. ${ }^{24,25}$

As discussed in Sec. 3.1, the PL emission of the binary P3HT:ADT-TES-F $(1: 1)$ blend was dominated by the P3HT/ADT-TES-F exciplex [Fig. 5(b) and Table 1]. Since the $J_{\text {sc }}$ was low in this blend, it follows that the P3HT/ADT-TES-F exciplex was inefficient in generating mobile charge carriers. Note that in spite of a very low $\triangle$ LUMO of $0.05 \mathrm{eV}$ in the P3HT:ADT-TES-F blend, the $J_{\mathrm{sc}}$ was comparable to that in the P3HT:ADT-TIPS-CN blend with a $\triangle$ LUMO of $0.49 \mathrm{eV}$, supporting our previous statement that $\triangle$ LUMO cannot predict the efficiency of photoinduced charge separation. ${ }^{37,43,44}$ A considerably higher $V_{\text {oc }}$ was expected from the binary P3HT: ADT-TES-F blend as compared to that in the P3HT:ADT-TIPS-CN blend, based on the LUMO energies of the ADT-TES-F and ADT-TIPS-CN (Fig. 1), but this was not observed (Table 1). Since the exciplex-forming properties of these two derivatives with P3HT were similar (Table 1), recombination of exciplex states ${ }^{39}$ could not account for the observed $V_{\text {oc }}$ differences. Therefore, in order to gain further insight into these observations, we removed the P3HT and explored binary SMBHJ solar cells with ADT-TES-F as the only donor.

\subsubsection{Small-molecule D:A systems-ADT-TES-F:PCBM and ADT-TES-F:ADT-TIPS-CN}

Solar cell characteristics of several D:A blends with ADT-TES-F as the donor are summarized in Table 2. In pristine ADT-TES-F diodes, the $V_{\text {oc }}$ of $0.95 \mathrm{~V}$ was consistent with the built-in voltage caused by differences in the work functions of $\mathrm{Al}(4.1 \mathrm{eV})$ and ITO/PEDOT:PSS $(5.1 \mathrm{eV})$. The $J_{\text {sc }}$ of $0.011 \mathrm{~mA} / \mathrm{cm}^{2}$, only slightly lower than $0.016 \mathrm{~mA} / \mathrm{cm}^{2}$ in P3HT:ADT-TES-F $(1: 1)$ and P3HT:ADT-TIPS-CN (1:1) blends, was observed (Table 2). This confirms that in the P3HT: ADT-TES-F $(1: 1)$ blend, most charge carriers are generated in separate P3HT and ADTTES-F domains rather than at the P3HT/ADT-TES-F interfaces. In ADT-TES-F:ADT-TIPSCN $(1: 1)$ and ADT-TES-F:PCBM $(1: 1)$ blends, the $J_{\text {sc }}$ increased by a factor of $\sim 2$ and of $\sim 50$, leading to improvements in PCEs by a factor of $\sim 2$ and $\sim 10$, respectively, as compared to pristine ADT-TES-F films. In the ADT-TES-F:ADT-TIPS-CN (1:1) blend, which had a considerably broader absorption spectrum than pristine ADT-TES-F [Fig. 4(a)], a relatively small improvement in the $J_{\mathrm{sc}}$ with respect to that in pristine ADT-TES-F films was due to the formation of a highly emissive exciplex [Fig. 4(b)], which has been shown to be detrimental for charge photogeneration efficiency. ${ }^{21,23,26,36}$ In the ADT-TES-F:PCBM blends, a considerably larger improvement in $J_{\mathrm{sc}}$ was observed (Table 2), most likely due to both enhanced charge photogeneration efficiency, previously observed in similar blends, ${ }^{25,28}$ and improved electron mobility. However, the $J_{\mathrm{sc}}$ of $0.52 \mathrm{~mJ} / \mathrm{cm}^{2}$ in the ADT-TES-F:PCBM $(1: 1)$ blend was more than an order of magnitude lower than that in the P3HT:PCBM $(1: 1)$ blend, in part due to the ADT-TES-F: PCBM film structure featuring crystalline ADT-TES-F domains with the orientations not favorable for vertical charge transport (Fig. 3). ${ }^{25}$ The FF was relatively low in all ADT-TES-F-containing SMBHJs, in part due to the poor quality of interface between the crystalline ADT-TES-F domains and the electrodes.

In contrast to $J_{\mathrm{sc}}$, which showed predictable trends upon ADT-TIPS-CN or PCBM acceptor addition to ADT-TES-F, the behavior of $V_{\text {oc }}$ was more intriguing. In the ADT-TES-F:ADTTIPS-CN $(1: 1)$ blend, the $V_{\text {oc }}$ was $0.77 \mathrm{~V}$, similar to $0.8 \mathrm{~V}$ in the P3HT:ADT-TIPS-CN (1:1) blend. This result is consistent with the relative HOMO and LUMO energies of ADTTES-F, P3HT, and ADT-TIPS-CN molecules (Fig. 1) and does not seem to be affected by the presence ${ }^{32}$ of the ADT-TES-F/ADT-TIPS-CN exciplex at $1.8 \mathrm{eV}$ in the ADT-TES-F: ADT-TIPS-CN $(1: 1)$ blend or the P3HT/ADT-TIPS-CN exciplex at $1.4 \mathrm{eV}$ in the P3HT: ADT-TIPS-CN (1:1) blend (Table 1). However, in ADT-TES-F:PCBM (1:1) blends, a $V_{\text {oc }}$ of $0.15 \mathrm{~V}$ was observed (Table 2), considerably lower than the $V_{\text {oc }}$ of $0.6 \mathrm{~V}$ obtained in P3HT:PCBM blends.

To gain further insight into the unexpectedly low $V_{\text {oc }}$ in ADT-TES-F:PCBM blends, we compared the time-resolved charge carrier dynamics of ADT-TES-F:PCBM blends with those of pristine ADT-TES-F and ADT-TES-F:ADT-TIPS-CN films, which we have studied in detail 


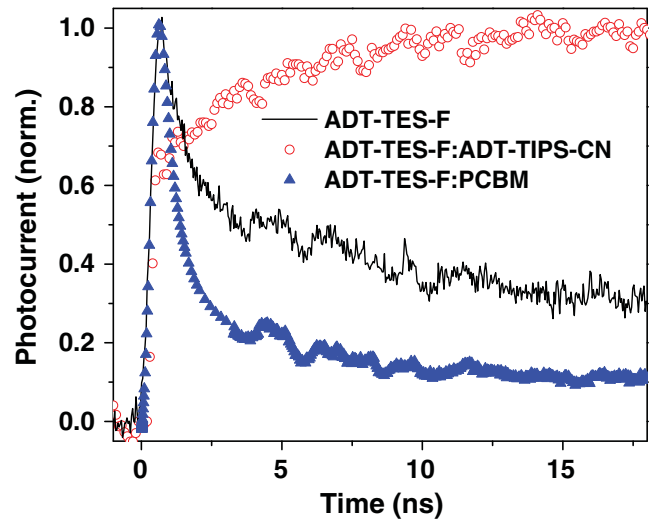

Fig. 10 Normalized transient photocurrents obtained in pristine ADT-TES-F films and ADT-TESF:PCBM and ADT-TES-F:ADT-TIPS-CN blends upon pulsed photoexcitation.

in our previous work..$^{20,21,23-26,28,36}$ In particular, the $V_{\text {oc }}$ has been shown to depend on the mechanism and efficiency of nongeminate recombination, ${ }^{45,46}$ which can be elucidated by analyzing photocurrent dynamics. ${ }^{28}$ Figure 10 shows normalized time-resolved photocurrents obtained under a pulsed $470 \mathrm{ps}, 532 \mathrm{~nm}$ excitation of pristine ADT-TES-F and ADT-TES-F:PCBM (9:1) films from Ref. 25 and under a pulsed $100 \mathrm{fs}, 400 \mathrm{~nm}$ excitation of ADT-TES-F: ADT-TIPS-CN $(9: 1)$ films from Ref. 36. In these measurements, a fast rise of the photocurrent, limited by the laser pulse width $(<0.5 \mathrm{~ns})$ and by the resolution of the detection system $(<30 \mathrm{ps})$, respectively, was observed, indicative of fast photogeneration of mobile charge carriers. The photocurrent dynamics at times longer than the pulse width incorporate charge carrier loss due to trapping and recombination. ${ }^{28}$ Numerical simulations of photocurrent dynamics in pristine ADT-TES-F films and in several ADT-TES-F-based blends have identified bimolecular recombination as the dominant process behind the sub-ns charge carrier loss observed via fast photocurrent decay in the ADT-TES-F film and in the ADT-TES-F:PCBM blend in Fig. $10 .^{25}$ Drastically different photocurrent dynamics observed in ADT-TES-F: PCBM and ADT-TES-F:ADT-TIPS-CN blends are indicative of enhanced and reduced fast bimolecular recombination, respectively, as compared to pristine ADT-TES-F films under the same excitation conditions. $^{25,36}$ Based on these observations, fast bimolecular recombination in ADT-TES-F: PCBM blends could be one of the reasons behind the loss of $V_{\text {oc }}$ in these blends. Note that the presence of the ADT-TES-F/ADT-TIPS-CN exciplex, which is the dominant photoinduced interaction between ADT-TES-F and ADT-TIPS-CN in binary blends, ${ }^{23}$ is not directly related to reduced charge carrier recombination; however, charge carrier recombination dynamics in $\mathrm{BHJ}$ depend considerably on the morphology of the D/A interfaces, which also determines exciplex properties. ${ }^{21}$ Numerical modeling of transient photocurrents similar to those in Fig. 10 revealed up to a factor of four enhancement in ultrafast charge carrier separation efficiency in ADT-TESF:PCBM (9:1) films as compared to pristine ADT-TES-F films, ${ }^{25}$ which is in trend with the $J_{\text {sc }}$ values in ADT-TES-F:PCBM SMBHJs in Table 2. A factor of $\sim 3$ decrease in the transient photocurrent amplitude in ADT-TES-F:ADT-TIPS-CN (9:1) films as compared to those in pristine ADT-TES-F films was attributed to inefficient charge photogeneration from exciplex states, ${ }^{36}$ also consistent with the $J_{\mathrm{sc}}$ trends in exciplex-forming blends in Table 2.

The ADT-TES-F:PCBM $(1: 1)$ blend exhibited an order of magnitude higher PCE as compared to that in either P3HT:ADT-TIPS-CN $(1: 1)$ or P3HT:ADT-TES-F $(1: 1)$ blends, in spite of the considerably lower $V_{\text {oc }}$ in the ADT-TES-F:PCBM BHJ (Table 2). Therefore, it appears that when there is a trade-off between achieving high $V_{\text {oc }}$ (e.g., due to enhanced recombination) and maximizing $J_{\mathrm{sc}}$ (e.g., due to enhanced charge photogeneration efficiency), the latter route is more productive for the PCE. ${ }^{42}$

\section{Conclusions}

Addition of a nonfullerene small molecule acceptor ADT-TIPS-CN to "efficient" P3HT:PCBM BHJs led to a reduction in $J_{\mathrm{sc}}, V_{\mathrm{oc}}$, and PCE, mostly due to decreased charge photogeneration 
Rajesh et al.: Design of organic ternary blends and small-molecule bulk heterojunctions...

efficiency and increased disorder. By contrast, addition of the small molecule donor ADT-TES-F to "inefficient" P3HT:ADT-TIPS-CN BHJs led to a factor of 2 to 3 improvement in $J_{\text {sc }}$, which resulted in a factor of 2 to 4 improvement in PCE. In particular, ternary P3HT:ADT-TES-F:ADTTIPS-CN $(1: x: 1)$ blends with $x=0.02$ to 0.5 exhibited better PCEs than those in the P3HT: ADT-TES-F (1:1), P3HT:ADT-TIPS-CN (1:1), or ADT-TES-F:ADT-TIPS-CN $(1: 1)$ binary blends. In all blends, exciplex formation did not reduce the $V_{\mathrm{oc}}$, but was correlated with a reduced $J_{\mathrm{sc}}$. Therefore, morphologies leading to efficient intermixing between various components of the blend, which promote exciplex formation, must be avoided. A factor of $\sim 5$ higher PCE was observed in ADT-TES-F:PCBM $(1: 1)$ as compared to ADT-TES-F:ADT-TIPS-CN (1:1) SMBHJs largely due to enhanced charge carrier photogeneration in the blend with PCBM.

In all blends containing the ADT-TES-F derivative, the observed $V_{\text {oc }}$ did not show any correlation with expectations based on the HOMO and LUMO energies of Fig. 1. The applicability of HOMO and LUMO energies measured in solution using cyclic or differential pulse voltammetry to solar cell analysis has been questioned in the literature. ${ }^{37,43,44}$ The importance of including ionization potentials and activation energies of optically excited states $^{43}$ and of local dielectric environment ${ }^{44}$ into consideration has been emphasized. While further studies are needed to conclusively establish the dominant loss factor that determines the $V_{\text {oc }}$ in our blends, our results clearly highlight the importance of the underlying photophysics, which goes far beyond HOMO/LUMO energy considerations, for solar cell characteristics.

\section{Acknowledgments}

This work was supported by University Grants Commission, India via Indo-US Raman Postdoctoral Fellowship- F.No. 5-50/2013(IC) and the National Science Foundation grant DMR-1207309. J.E.A. and R.H. thank the National Science Foundation (CMMI-1255494) for support of the synthesis of organic semiconductors.

\section{References}

1. M. C. Scharber and N. S. Sariciftci, "Efficiency of bulk-heterojunction organic solar cells," Prog. Polym. Sci. 38, 1929 (2013).

2. J. Servaites, M. A. Ratner, and T. J. Marks, "Organic solar cells: a new look at traditional models," Energy Environ. Sci. 4, 4410 (2011).

3. O. Ostroverkhova, ed., Handbook of Organic Materials for Optical and (Opto)electronic Devices, p. 800, Woodhead Publishing, Oxford (2013).

4. S. Dimitrov and J. R. Durrant, "Materials design considerations for charge generation in organic solar cells," Chem. Mater. 26, 616 (2014).

5. S. Gelinas et al., "Ultrafast long-range charge separation in organic semiconductor photovoltaic diodes," Science 343, 512 (2014).

6. E. Bittner and C. Silva, "Noise-induced quantum coherence drives photo-carrier generation dynamics at polymeric semiconductor heterojunctions," Nat. Commun. 5, 3119 (2014).

7. Heliatek, http://www.heliatek.com/ (2014).

8. A. Troisi, "What makes fullerene acceptors special as electron acceptors in organic solar cells and how to replace them," Adv. Mater. 25, 1038 (2013).

9. J. E. Anthony, "Small-molecule, nonfullerene acceptors for polymer bulk heterojunction organic photovoltaics," Chem. Mater. 23, 583-590 (2011).

10. J. Roncali, "Molecular bulk heterojunctions: an emerging approach to organic solar cells," Acc. Chem. Res. 42, 1719-1730 (2009).

11. B. Walker, C. Kim, and T. Q. Nguyen, "Small molecule solution-processed bulk heterojunction solar cells," Chem. Mater. 23, 470-482 (2011).

12. Y. Lin, Y. Li, and X. Zhang, "Small molecule semiconductors for high-efficiency organic photovoltaics," Chem. Soc. Rev. 41, 4245 (2012).

13. A. Mishra and P. Bauerle, "Small molecule organic semiconductors on the move: promises for future solar energy technology," Angew. Chem.-Int. Ed. 51, 2020-2067 (2012). 
Rajesh et al.: Design of organic ternary blends and small-molecule bulk heterojunctions...

14. V. Gupta et al., "Barium: an efficient cathode layer for bulk heterojunction solar cells," Sci. Rep. 3, 1965 (2013).

15. Y. Liu et al., "Solution-processed small-molecule solar cells: breaking the $10 \%$ power conversion efficiency," Sci. Rep. 3, 3356 (2013).

16. T. Ameri et al., "Organic ternary solar cells: a review," Adv. Mater. 25, 4245 (2013).

17. R. A. Street et al., "Origin of the tunable open-circuit voltage in ternary blend bulk heterojunction organic solar cells," J. Am. Chem. Soc. 135, 986 (2013).

18. C. Proctor, M. Kuik, and T. Q. Nguyen, "Charge carrier recombination in organic solar cells,” Prog. Polym. Sci. 38, 1941 (2013).

19. J. Huang et al., "Polymer bulk heterojunction solar cells employing FRET," Nat. Photonics 7, 479 (2013).

20. A. D. Platt et al., "Optical, fluorescent, and photoconductive properties of high-performance functionalized pentacene and anthradithiophene derivatives," J. Phys. Chem. C 113, 1400614014 (2009).

21. M. J. Kendrick et al., "Formation of the donor-acceptor charge transfer exciton and its contribution to charge photogeneration and recombination in small-molecule bulk heterojunctions," J. Phys. Chem. C 116, 18108 (2012).

22. S. Subramanian et al., "Chromophore fluorination enhances crystallization and stability of soluble anthradithiophene semiconductors," J. Am. Chem. Soc. 130, 2706-2707 (2008).

23. W. E. B. Shepherd et al., "Energy transfer and exciplex formation and their impact on exciton and charge carrier dynamics in organic films," J. Phys. Chem. Lett. 2, 362-366 (2011).

24. K. Paudel et al., "Small-molecule bulk heterojunctions: distinguishing between effects of energy offsets and molecular packing on optoelectronic properties," Jo. Phys. Chem. C 117, 24752 (2013).

25. K. Paudel et al., "Enhanced charge photogeneration promoted by crystallinity in small-molecule donor-acceptor bulk heterojunctions," Appl. Phys. Lett. 105, 043301 (2014).

26. A. D. Platt et al., "Temperature dependence of charge carrier and exciton dynamics in organic thin films," Phys. Rev. B 84, 235209 (2011).

27. W. E. B. Shepherd et al., "Aggregate formation and its effect on (opto)electronic properties of guest-host organic semiconductors," Appl. Phys. Lett. 97, 163303 (2010).

28. B. Johnson, M. J. Kendrick, and O. Ostroverkhova, "Charge carrier dynamics in organic semiconductors and their donor-acceptor composites: numerical modeling of time-resolved photocurrent," J. Appl. Phys. 114, 094508 (2013).

29. O. D. Jurchescu et al., "Effects of polymorphism on charge transport in organic semiconductors," Phys. Rev. B 80, 085201 (2009).

30. O. D. Jurchescu et al., "Organic single-crystal field-effect transistors of a soluble anthradithiophene," Chem. Mater. 20(21), 6733-6737 (2008).

31. S. K. Park et al., "High-mobility spin-cast organic thin film transistors," Appl. Phys. Lett. 93, 043301 (2008).

32. D. J. Gundlach et al., "Contact-induced crystallinity for high-performance soluble acenebased transistors and circuits," Nat. Mater. 7, 216-221 (2008).

33. A. Pearson et al., "Rationalizing phase transitions with thermal annealing temperatures for P3HT:PCBM organic photovoltaic devices," Macromolecules 45, 1499 (2012).

34. T. Erb et al., "Correlation between structural and optical properties of composite polymer/ fullerene films for organic solar cells," Adv. Funct. Mater. 15, 1193 (2005).

35. A. D. Platt et al., "Photophysical and photoconductive properties of organic semiconductor composites," Proc. SPIE 7413, 7413-7428 (2009).

36. J. Day et al., "Organic semiconductor composites: influence of additives on the transient photocurrent," Appl. Phys. Lett. 94(1), 013306 (2009).

37. D. Veldman, S. C. J. Meskers, and R. A. J. Janssen, "The energy of charge-transfer states in electron donor-acceptor blends: insight into the energy losses in organic solar cells," Adv. Funct. Mater. 19, 1939 (2009).

38. S. Cook, A. Furube, and R. Katoh, "Analysis of the excited states of regioregular polythiophene P3HT," Energy Environ. Sci. 1, 294 (2008).

39. C. Deibel, T. Strobel, and V. Dyakonov, "Role of the charge transfer state in organic donoracceptor solar cells," Adv. Mater. 22, 4097-4111 (2010). 
40. T. Ripolles, A. Guerrero, and G. Garcia-Belmonte, "Polymer defect states modulate opencircuit voltage in bulk heterojunction solar cells," Appl. Phys. Lett. 103, 243306 (2013).

41. B. Qi and J. Wang, "Fill factor in organic solar cells," Phys. Chem. Chem. Phys. 15, 8972 (2013).

42. B. Minnaert and M. Burgelman, "Empirical study of the characteristics of current-state organic bulk heterojunction solar cells," Eur. Phys. J. Appl. Phys. 38, 111 (2007).

43. X. Y. Zhu, "How to draw energy level diagrams in excitonic solar cells," J. Phys. Chem. Lett. 5, 2283 (2014).

44. S. Chen et al., "Dielectric effect on the photovoltage loss in organic photovoltaic cells," Adv. Mater. 26, 6125 (2014).

45. Z. Beiley et al., "Morphology-dependent trap formation in high performance polymer bulk heterojunction solar cells," Adv. Energy Mater. 1, 954 (2011).

46. D. Credgington et al., "Non-geminate recombination as the primary determinant of opencircuit voltage in polythiophene:fullerene blend solar cells: an analysis of the influence of device processing conditions," Adv. Funct. Mater. 21, 2744 (2011).

Biographies of the authors are not available. 